\title{
Assessing the simulation of chaotic systems
}

\author{
F. J. BREMNER and M. YOST \\ Trinity University, San Antonio, Texas
}

\begin{abstract}
Three commercially available software packages-BrainMaker, MicroSaint, and Extend-that simulate the function and operation of neural networks were compared on 11 dimensions. Also, a task-oriented analysis was done on the ability of the programs to use a neural network to output a chaotic array generated by the logistic equation. There were differences in the amount of operator overhead among the programs, but the differences in the simulation performance were not considered to be appreciable.
\end{abstract}

In this paper, we compare three commercially available simulation programs in terms of a number of characteristics that fall under the general rubric of user friendliness. In addition to the standard table of items for evaluating programs (ease of recovery, readability of manual, use of menus, reliability, computing power, cost, etc.), the authors used a task-oriented strategy to compare the programs. The three programs are MicroSaint (1987), BrainMaker (1988), and Extend (1989). The task with which these programs were tested was to output an array from the chaotic equation called the logistic (May, 1976). However, before examining this unique approach to program evaluation, we will present an assessment of the standard characteristics of the programs.

MicroSaint, BrainMaker, and Extend are all readily available, have been in the field for a number of years, and have logged many hours of use. Two of the programs (MicroSaint, BrainMaker) operate in either the Macintosh or the DOS environments, but only their DOS versions will be discussed here. Extend, on the other hand, operates only in the Macintosh environment and uses the typical Macintosh operator interface of pull-down menus and mouse clicking. In this regard, all three programs are menu driven and capitalize on fast clocks and math coprocessor chips. All three programs were rated as excellent on these dimensions. A summary of the characteristics of each program is given in Table 1 .

To evaluate the quality of a computer program, it is important to know how it will be used. If, for example, a program is to serve primarily as a teaching aid, computing power is not as important as user friendliness. On the other hand, if the program is to be used in "state-of-the-art" research, computer power and machine overhead are as important as friendliness. In the teaching environment, there are considerable contrasts among the programs. BrainMaker is available in three versions, one of which, the Student Version, is much less powerful than the Profes-

Correspondence should be addressed to Frederick J. Bremner, Department of Psychology. Trinity University, 715 Stadium Drive, San Antonio, TX 78212 sional Version, but they are equally user friendly. MicroSaint is available in two versions, one of which is a student version; however, we will be reviewing the standard version of MicroSaint. Although MicroSaint is user friendly, it is considerably more difficult to use than BrainMaker. Extend is also more difficult to use than BrainMaker, but it is very powerful and strictly adheres to the typical Macintosh user interface. Therefore, the typical Macintosh user can quickly become familiar with its operations. An exercise could be prepared in Extend's scripting language and "canned" so that students could put the iconic modules together. With this approach, Extend would be an excellent way for undergraduates to learn about neural networks. We have used these programs extensively with undergraduate students without any major difficulty. We and our undergraduate students learned to use the programs from the manuals without receiving any special instruction.

The Student Version of BrainMaker is not much more than a demonstration program, and we have found it to be too underpowered for undergraduate classes. Nevertheless, California Scientific should be commended for its packaging of the Student Version. For $\$ 20.00$, students receive an introductory text, the standard computer manual, and a floppy disk with the Student Version of the program on it. However, the authors feel that a license arrangement for a more powerful version should be part of the standard classroom package. For the remainder of this paper, the intermediate version of BrainMaker will be the version of that program reviewed.

The major contrast among the programs is that MicroSaint and Extend use the production system strategy (Boden, 1988) in their simulations, whereas BrainMaker uses the backpropagation strategy (Lawrence, 1988). Because of the user interface, Extend has an iconic mode of operation that makes the production system strategy less apparent with this program. In short, if one intends to teach a production system method of simulation, MicroSaint would be the program of choice. On the other hand, this program is probably the most difficult of the three to use. One suspects that general simulation programs such as MicroSaint and Extend, using the production system 
Table 1

\begin{tabular}{|c|c|c|c|}
\hline & \multicolumn{3}{|c|}{ Program } \\
\hline & MicroSaint & BrainMaker & Extend \\
\hline$\overline{\text { Computing Power }}$ & Excellent & Good & Excellent \\
\hline Applicability & Broad and flexible & Flexible & $\begin{array}{l}\text { Extremely flexible } \\
\text { with a wide } \\
\text { range of applications }\end{array}$ \\
\hline Communication & Menu driven & $\begin{array}{l}\text { Single screen, } \\
\text { menu driven }\end{array}$ & $\begin{array}{l}\text { Menu driven, } \\
\text { multiple windows: } \\
\text { adheres well to } \\
\text { Macintosh interface }\end{array}$ \\
\hline Strategy & Production system & Backpropagation & Production system \\
\hline Ease of Recovery & Excellent & Good & Excellent \\
\hline Manual & $\begin{array}{l}\text { Readable; an } \\
\text { example using } \\
\text { psychological variables } \\
\text { would help }\end{array}$ & $\begin{array}{l}\text { Readable; needs } \\
\text { some proofreading } \\
\text { for typos }\end{array}$ & $\begin{array}{l}\text { Readable; tends } \\
\text { to cater to } \\
\text { business application } \\
\text { organization is a } \\
\text { little confusing }\end{array}$ \\
\hline Reliability & Excellent & Excellent & Excellent \\
\hline Environment & DOS/Macintosh & DOS/Macintosh & Macintosh \\
\hline Drawback & High machine overhead & Processing speed & $\begin{array}{l}\text { High overhead; } \\
\text { few global variables } \\
\text { (can be overcome } \\
\text { with use of arrays) }\end{array}$ \\
\hline Cost (after discount) & $\$ 2,232.00$ & $\$ 156.00$ & $\$ 297.00$ \\
\hline Vendor & $\begin{array}{l}\text { Micro Analysis and } \\
\text { Design, Inc. } \\
3300 \text { Mitchell Lane } \\
\text { Suite } 175 \\
\text { Boulder, CO } 80301 \\
\text { (303) } 442-6947 \\
\text { FAX } 303-442-8274\end{array}$ & $\begin{array}{l}\text { California Scientific Software } \\
10024 \text { Newtown Rd. } \\
\text { Nevada City, CA } 95959 \\
\text { (916) 478-9040 } \\
\text { FAX } 916-477-8656\end{array}$ & $\begin{array}{l}\text { Imagine That, Inc. } \\
\text { 151 Bernal Rd., } \\
\text { Suite 5 } \\
\text { San Jose, CA } 95119 \\
\text { (408) 365-0305 }\end{array}$ \\
\hline
\end{tabular}

strategy, would have a broader application than would programs using the backpropagation strategy. The manuals for MicroSaint and Extend seem to reflect a wider range of applications than does the BrainMaker manual. However, in a more creative sense, the applicability really depends on the ability and experience of the user. In fact, we used the MicroSaint program to do backpropagation during the testing of programs for this report, and the BrainMaker manual contains an example of a process control system. Nevertheless, the two approaches are considerably different.

In the production system approach, one relies on a series of "if-then-else" statements to drive the simulation, while the dynamics of the system are controlled by the functions assigned to the variables. For example, one could say that if a random number between $x$ and $y$ occurs, then the next step in the simulation happens. In backpropagation, the collective synaptic weights of the neurons determine whether the next step in the simulation occurs (Lawrence, 1988). These synaptic weights are assigned in accordance with the Hebbian learning rule
(McClelland \& Rumelhart, 1988); therefore, BrainMaker learns the best solution. Although the Extend program uses a production system strategy, the iconic style of the Macintosh masks it. With Extend, one can do either production system simulation or, as we did here, backpropagation simulation. We believe that the best way to compare the different simulation strategies is to have the programs simulate the same task. The remainder of this paper is dedicated to doing just that.

The students who worked on the task-oriented evolution of the project were undergraduates who were majoring or minoring in psychology. They were enrolled in lower division psychology courses at a predominately liberal arts and science institution in the Southwest. The hardware in all of the tests comprised an MS-DOS-compatible, 80386 personal computer (for BrainMaker and MicroSaint) and a Macintosh II (for Extend). The software comprised Version 3.2 of the MicroSaint program by Micro Analysis and Design, BrainMaker Version 2.2 by Califomia Scientific, and Extend Version 1.1 by Imagine That. Procedurally, the logistic equation was programmed with the 


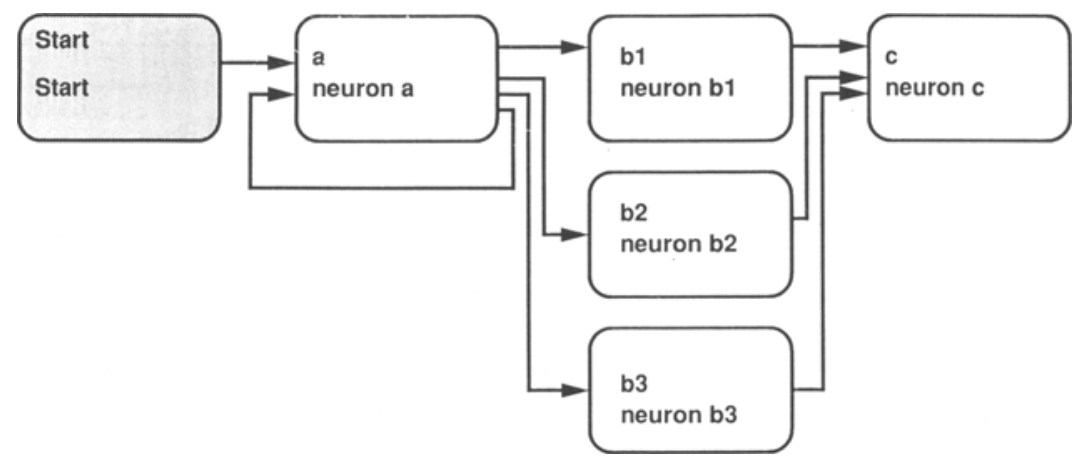

Figure 1. Typical diagram of a backpropagation 1-4-1 neural network. The start node sets initial conditions and is not part of the iterative process.

high-order language of the three commercially available programs. In BrainMaker, a backpropagation net was designed that had three layers. There were six neurons in all. Layer 1 had one neuron, Layer 2 had four neurons, and Layer 3 had one neuron. This is the smallest network that would nun the logistic equation. In MicroSaint, a threelayer network was also designed that had one input neuron, three middle neurons, and one output neuron. There was one other node in the network (START) that set the initial conditions. Using the Macintosh interface and the iconic strategy of the Extend program, a backpropagation neural network was designed. In addition to the neurons, a preprocessing block was used to set initial conditions and parameters. Once these networks were built, several inputs were tested to determine the functioning of the network. These inputs involved changing the constant of the logistic equation. (See Figure 1.)

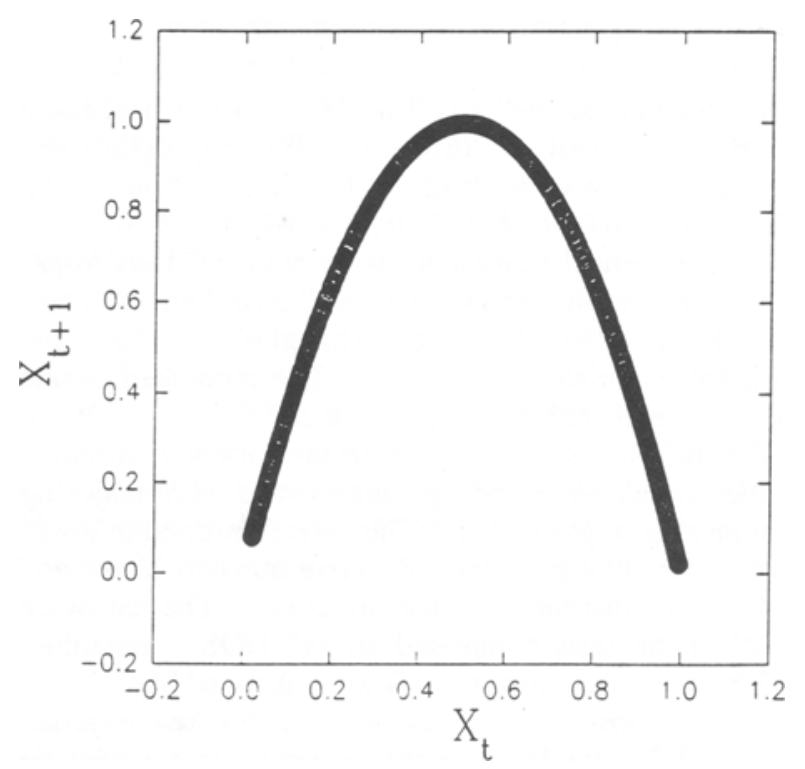

Figure 2. Typical form of the output of the logistic equation when $X$ is plotted against $X+1$ (i.e., self-phase-portrait).
The strategy employed was to test the simulation programs on the logistic equation $k(1-X n) X n$ to determine user task difficulty in working with each program. This equation is nonlinear, and when implemented, it will cause the data to vacillate wildly on some occasions and to appear regular on other occasions. Graphic output of this equation has previously been published (May, 1976). It is believed that many natural phenomena are chaotic. Such things as the weather, neuron firing patterns (Mpitsos, Burton, Creech, \& Soinila, 1988), and animal populations (May, 1976) have been shown to be chaotic. An example of the phase portrait generated by the logic equation is given in Figure 2.

In 1988, Mpitsos and others (Mpitsos, Burton, \& Creech, 1988) demonstrated that neural networks could be programmed to learn and transmit chaotic signals. All three of the programs tested will transmit chaos of the logistic equation type, and the data analysis of their output produces the typical hyperbola associated with the logistic form of chaos. These results support previous work on the ability of neural networks to learn and transmit chaos (Mpitsos, Burton, \& Creech, 1988). Figure 2 represents the output of the MicroSaint program. This output is not different from that of BrainMaker or Extend. The time to generate each network was shorter for BrainMaker, but this was probably due to the students' having done a similar exercise previously and the fact that it is inherently a backpropagation program. There was very little difference in the labor involved in building the neural networks with the three programs described here.

The students who participated in this project felt that the assignment they were given was reasonable, and most were able to complete it with only minimal interaction with the professor. Of course the authors and students understood the strategies underlying each program before beginning this project. Since an "if-then-else" strategy is very different from a backpropagation strategy, it is necessary for the user to conceive the problem in the appropriate strategy. This task was picked because the instructors could readily conceive it with either strategy. Some problems would favor one strategy over the other. 
If the task were to determine the number of personnel necessary to operate a chemical decontamination center in Kuwait, the authors would opt for MicroSaint. On the other hand, if the problem were to adjust robotic spray guns to paint coffee cups and tea cups differently, BrainMaker would be the program of choice (Arthur, 1990). Extend's being a general simulation program would make it a good choice to do either task, given a Macintosh user of some sophistication. In summary, the outcome of this project was to remind the authors that, as Marr (1982) advocated, a computational (or task) analysis needs to be done before the simulation is started.

\section{REFERENCES}

ARTHUR, B. (1990, April). Back to backpropagation on the Mac. Paper presented at the meeting of the Southwest Psychological Association, Dallas.
Boden, M. A. (1988). Computer models of the mind. New York: Cambridge University Press.

BrainMaker [Computer program] (1988). Nevada City, CA: California Scientific Software.

Extend [Computer program] (1989). San Jose, CA: Imagine That, Inc. LAWRENCE, J. (1988). Introduction to neural networks. Nevada City, CA: California Scientific Software.

MAY, R. M. (1976). Simple mathematical models with very complicated dynamics. Nature, 261, 459-467.

McClelland, J. L., \& Rumelhart, D. E. (1988). Explorations in parallel distributed processing. Cambridge, MA: MIT Press.

MARR, D. (1982). Vision: A computational investigation into the human representation and processing of visual information. San Francisco: W. H. Freeman.

MicroSaint [Computer program] (1987). Boulder, CO: Micro Analysis \& Design, Inc.

Mpitsos, G. J., Burton, R. M., Creech, H. C. (1988). Connectionist networks learn to transmit chaos. Brain Research, 21, 539-546.

Mpitsos, G. J., Burton, R. M., Creech, H. C., \& Soinila, S. O. (1988). Evidence for chaos in spike trains of neurons that generate rhythmic motor patterns. Brain Research, 2, 529-538. 